Publisher: Taylor \& Francis

Journal: International Journal of Control

DOI: https://doi.org/10.1080/00207179.2017.1408923

\title{
BOOSTING THE DECAY OF SOLUTIONS OF THE LINEARIZED KORTEWEG-DE VRIES-BURGERS EQUATION TO A PREDETERMINED RATE FROM THE BOUNDARY
}

\author{
TÜRKER ÖZSARI* \\ Department of Mathematics, Izmir Institute of Technology, Urla, Izmir, 35430 TUKKEY \\ EDA ARABACI \\ Department of Mathematics, Izmir Institute of Technology, Uriw, Izmir, 35430 TURKEY
}

\begin{abstract}
The aim of this article is to extend recent results on the boundary feedback controllability of the Korteweg-de Vries equation (KdV) to the Korteweg-de Vries-Burgers equation (KdVB) which is posed on a bounded domain. In the first part of the paper, it is proven that all sufficiently small solutions can be steered to zero at any desired exponential rate by means of a suitably constructed boundar feedbazk controller. In the second part, an observer is proposed when a type of boundary reasurement is available while there is no full access to the medium.
\end{abstract}

\section{Contents}

1. Introduction

2. Controller Design

2.1. Linearized equation 4

2.2. Nonlinear Stability 10

3. Observer design 11

3.1. Linearized equation 11

3.2. Other boundary conditions 14

References 16

E. mail addresses: tozsari@gmail.com, edaarabaci@iyte.edu.tr.

2010 Marhematics Subject Classification. 93D15, 35Q53, 93C20.

Key words and phrases. Korteveg-de-Vries Burgers equation; back-stepping; feedback stabilization;

bour dary controller; and observer design.

*Corresponding author: tozsari@gmail.com.

twhe results of this paper will also appear as part of the M.S. thesis of Eda Arabac1. 


\section{INTRODUCTION}

This article is devoted to the study of the boundary feedback controllability of the Kortewegde Vries Burgers equation (KdVB), which is posed on a bounded interval, say $\Omega=(0,1)$ :

$$
\begin{cases}u_{t}-u_{x x}+u_{x x x}+u u_{x}=0 & \text { in } \Omega \times \mathbb{R}_{+}, \\ u(0, t)=U(t), u(1, t)=u_{x}(1, t)=0 & \text { in } \mathbb{R}_{+}, \\ u(x, 0)=u_{0}(x) & \text { in } \Omega .\end{cases}
$$

In (1.1), $u=u(x, t)$ is a real valued function, which can for example model the evolution of the amplitude of a long shallow water wave in space and time. The input $U(t)=J(\varphi(t, \cdot))$ at the left end point of the boundary is a feedback, which will be appropriately constructed by using the back-stepping method (see e.g., (Krstic \& Smyshlyaev, 200s 1 to steer the solutions of (1.1) to zero as $t \rightarrow \infty$, at a predetermined exponential rate of decay in a physically meaningful sense. More precisely, we study the following stabilization problem:

"Given $\lambda>0$, is there a boundary feedback $U(t)=U(u(t, \cdot))$ such that the solution of (1.1) satisfies $\|u(\cdot, t)\|_{L^{2}(\Omega)}=O\left(e^{-\lambda t}\right)$ ?"

The KdV-Burgers equation has an intrinsic decay property. For instance, (Amick et al., 1989) shows that if $u$ is a solution of the Cauchy problem for the KdV Burgers equation on $\mathbb{R}$, then

$$
\|u(t)\|_{L^{2}(\mathbb{R})}^{2}=O\left(t^{-\frac{1}{2}}\right) \text {. }
$$

However, in the same study it is shown that this result is generally sharp and one should not expect a decay rate faster than the one given above.

The situation for the case of bounded domains is difrerent and one can get exponential decay of solutions with an ideal set of boundary conditions. For example, in the absence of the feedback $(U \equiv 0)$, multiplying (1.1) with a , integrating over $\Omega=(0,1)$, using the Poincaré inequality and the given bounaary conditions, one can see that (see also (Liu \& Krstić, 2002))

In other words,

$$
\left\|\operatorname{mat}_{1}(t)\right\|_{L^{2}(\Omega)} \leq\left\|u_{0}\right\|_{L^{2}(\Omega)} e^{-t}
$$

$$
\|n(\cdot, t)\|_{L^{2}(\Omega)}=O\left(e^{-t}\right) \text {. }
$$

If the dispersive effect is smail then the $\mathrm{KdV}$-Burgers equation is written

$$
u_{t}-\epsilon u_{x x}+u_{x x x}+u u_{x}=0,
$$

where $\epsilon>0$ is a mall number. In this case, the decay rate slows down and is given by

$$
\|u(t)\|_{L^{2}(\Omega)}=O\left(e^{-\epsilon t}\right) .
$$

Our aim is simpiy boosting the decay rate to $O\left(e^{-\lambda t}\right)$ for a prescribed $\lambda>0$. It is natural to enffree a feeáback controller into the system to ensure faster decay of solutions. There is already some work in this direction where the stabilization of the KdV-Burgers equation is strdied by means of various interior or boundary feedback laws. See for example (Cavalcanti et a .., 2014) for an internal controller designed to stabilize the solutions of KdV-Burgers equation. Although internal damping mechanisms are useful, sometimes it is difficult or impossible to access the medium of the evolution, in which case boundary controllers play a significant role. Some examples of such boundary control mechanisms for the KdV-Burgers or similar equations are given in (Balogh \& Krstic, 2000), (Liu \& Krstić, 2002), (Sakthivel, 2009), (Smaoui et al., 2010a), (Smaoui et al., 2010b), (Jia \& Zhang, 2012), and (Jia, 2016). 
However, in all of these studies, the boundary controllers are pre-configured, unlike a backstepping type controller.

In the last twenty years, there has been an abundance of interest and results in the subject of the controllability of KdV. Some notable papers in this subject are (Komornik et al., 1991), (Zhang, 1994), (Russell \& Zhang, 1996), (Rosier, 1997), (Zhang, 1999), (Rosier, 2000), (Bona et al., 2003), (Rosier \& Zhang, 2006), (Linares \& Pazoto, 2007), (Cerpa, 2007) (Massarolo et al., 2007), (Rosier \& Zhang, 2009), (Cerpa \& Crépeau, 2009), and (Laurent et al., 2010). Recently, (Cerpa \& Coron, 2013) studied the boundary feedback stabilization of the Korteweg-de Vries (KdV) equation

$$
u_{t}+u_{x}+u_{x x x}+u u_{x}=0
$$

with the same type of boundary conditions given in (1.1) by using the so-called back-stepping technique. (Cerpa \& Coron, 2013) proved that given any $\lambda>0$, one can find a boundary feedback controller $U(t)=U(u(t, \cdot))$ such that the solution of (1.2) satisties

$$
\|u(t)\|_{L^{2}(\Omega)} \lesssim\left\|u_{0}\right\|_{L^{2}(\Omega)} e^{-\lambda t}
$$

if $u_{0}$ is sufficiently small. We extend this result to the KdV-Burgers equation and we prove the following theorem for problem (1.1).

Theorem 1.3. Let $\lambda>0$. Then, there exists $\delta>0$ and $C^{3}$-kernel function $k=k(x, y)$ such that the solution of (1.1) subject to the boundary coniromer $U(t)=\int_{0}^{1} k(0, y) u(y, t) d y$ satisfies

$$
\|u(t)\|_{L^{2}(\Omega)} \lesssim e^{-\lambda t\left\|_{1} u_{0}\right\|_{12} L_{2}(\Omega)}
$$

for $t \geq 0$ whenever $\left\|u_{0}\right\|_{L^{2}(\Omega)}<\delta$.

Remark 1.5. (1) The smallness condition $L_{0} L_{2}(\Omega)<\delta$ in the above theorem comes from the nonlinear nature of the equatron (1). This assumption is not necessary for the linearized KdV-Burgers equation, see for example Proposition 2.28 in Section 2.1.

(2) One of the novelties of this paper is to give a rigorous proof of the kernel function used in the above thoorem.

In the second part of this paper, motivated by (Marx \& Cerpa, 2014), (Marx \& Cerpa, 2016), and (Hasan, 2015), we corisider the situation where the system is not fully observable and only partial intormation can be extracted from the original model. We first study the linearized KdVB equation below.

$$
\begin{cases}u_{t}-u_{x x}+u_{x x x}=0 & \text { in } \Omega \times \mathbb{R}_{+} \\ u(0, t)=U(t), u(1, t)=u_{x}(1, t)=0 & \text { in } \mathbb{R}_{+} \\ u(x, 0)=u_{0}(x) & \text { in } \Omega\end{cases}
$$

An observer is proposed for the above equation when it is not possible to fully access the medium. while partial information can be extracted, such as a measurement from one end of the boundary. More precisely, we will consider the following observer with a boundary measurement of type $y(t)=\partial_{x}^{2} u(1, t)$ :

$$
\left\{\begin{array}{l}
\hat{u}_{t}-\hat{u}_{x x}+\hat{u}_{x x x}+p_{1}(x)\left[y(t)-\partial_{x}^{2} \hat{u}(1, t)\right]=0, \\
\hat{u}(0, t)=U(t), \hat{u}(1, t)=\hat{u}_{x}(1, t)=0 \\
\hat{u}(x, 0)=\hat{u}_{0}(x)
\end{array}\right.
$$

We prove the $H^{3}$-stabilization below. 
Theorem 1.8. Let $\lambda>0$ and $k(x, y)$ be the $C^{3}$-kernel function obtained in Theorem 1.3. Then, there exists a function $p_{1}=p_{1}(x)$ such that the solution $(u, \hat{u})$ of the system (1.6)-(1.7) with the feedback controller $U(t)=\int_{0}^{1} k(0, y) \hat{u}(y, t) d y$ satisfies

$$
\|u-\hat{u}\|_{H^{3}(\Omega)}+\|\hat{u}\|_{L^{2}(\Omega)} \lesssim\left(\left\|u_{0}-\hat{u}_{0}\right\|_{H^{3}(\Omega)}+\left\|\hat{u}_{0}\right\|_{L^{2}(\Omega)}\right) e^{-\lambda t} .
$$

for $t \geq 0$.

One can alternatively consider other types of boundary conditions and measurements to design an observer. Consider for instance the linearized KdV-Burgers equation

$$
\begin{cases}u_{t}-u_{x x}+u_{x x x}=0 & \text { in } \Omega \times \mathbb{R}_{+}, \\ u(0, t)=U(t), u_{x}(1, t)=u_{x x}(1, t)=0 & \text { in } \mathbb{R}_{+}, \\ u(x, 0)=u_{0}(x) & \text { in } \Omega .\end{cases}
$$

If the above system were fully observable one could design a boundary fontroller in the form $U(t)=\int_{0}^{1} k(0, y) u(y, t) d y$, where $k(x, y)$ is a kernel function satisfying (3.27). If only partial measurement is available, say $y(t)=u(1, t)$, then one can use the observer given by:

$$
\left\{\begin{array}{l}
\hat{u}_{t}-\hat{u}_{x x}+\hat{u}_{x x x}+p_{1}(x)[y(t)-\hat{u}(1, t) \\
\hat{u}(0, t)=U(t), \hat{u}_{x}(1, t)=\hat{u}_{x x}(1, t)=0 \\
\hat{u}(x, 0)=\hat{u}_{0}(x) .
\end{array}\right.
$$

Indeed, we prove the following theorem for the above observer.

Theorem 1.12. Let $\lambda>0$ and $k(x, y)$ be the $C^{3}-k$ ernel function in (3.27). Then, there exists a $C^{3}$-function $p_{1}=p_{1}(x)$ such that the solition $\left.w, \hat{u}\right)$ of the system (1.10)-(1.11) with the feedback controller $U(t)=\int_{0}^{1} k(0, y) \hat{u}(y, t) d y$ satisfies

$$
\|u-\hat{u}\|_{L^{2}(\Omega)}+\|\hat{u}\|_{L^{2}(\Omega)} \lesssim\left(\left\|u_{0}-u_{0}\right\|_{L^{2}(\Omega)}+\left\|\hat{u}_{0}\right\|_{L^{2}(\Omega)}\right) e^{-\lambda t} .
$$

for $t \geq 0$.

\section{Controller Design}

2.1. Linearized equation. Qur goal is to transform (1.6) into the following homogeneous boundary value problem by means of an appropriate boundary controller $U(t)$ :

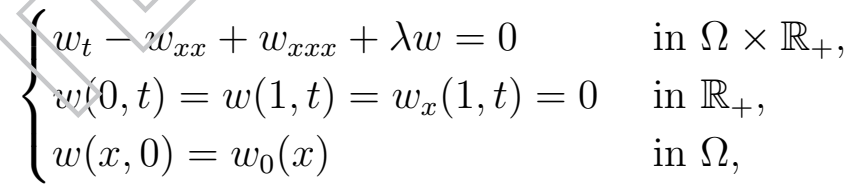

where $\lambda>0$. The reason is that the solution of (2.1) satisfies $\|w(t)\|_{L^{2}(\Omega)}=O\left(e^{-\lambda t}\right)$. In order to see ibis, multiply the main equation in (2.1) by $w$, integrate over $\Omega$, and use the given initial-boundary conditions to get

$$
\frac{1}{2} \frac{d}{d t}\|w(t)\|_{L^{2}(\Omega)}^{2}=-\left\|w_{x}(t)\right\|_{L^{2}(\Omega)}^{2}-\frac{1}{2}\left|w_{x}(0, t)\right|^{2}-\lambda\|w(t)\|_{L^{2}(\Omega)}^{2},
$$

from which it follows that

$$
\|w(t)\|_{L^{2}(\Omega)} \leq e^{-\lambda t}\left\|w_{0}\right\|_{L^{2}(\Omega)}
$$

for $t \geq 0$. In other words, solutions of (2.1) decay to zero exponentially fast. Therefore, we will refer to (2.1) as the damped equation and $\lambda$ as the damping coeffcient. 
To this end, we define the transformation

$$
w(x, t) \equiv u(x, t)-\int_{x}^{1} k(x, y) u(y, t) d y
$$

where the unknown kernel function $k(x, y)$ will be chosen in such a way that if $u$ is a solution of (1.6) with boundary feedback controller

$$
U(t) \equiv \int_{0}^{1} k(0, y) u(y, t) d y
$$

then $w$ is a solution of the homogeneous initial-boundary value problem (2.1) with initial datum

$$
w_{0}=u_{0}-\int_{x}^{1} k(x, y) u_{0}(y) d y
$$

Remark 2.6. Taking $L^{2}(\Omega)$-norms of both sides of (2.4), one can easily show that

$$
\|w(t)\|_{L^{2}(\Omega)} \lesssim\|u(t)\|_{L^{2}(\Omega)}
$$

where the constant of the inequality depends on the function $k$

In order to find the unknown kernel function $k$, we simply assure that $u$ solves (1.6) and plug in $u(x, t)-\int_{x}^{1} k(x, y) u(y, t) d y$ into the main equation in (2.1) wherever we see $w$. Note that $w$ satisfies the given homogeneous boundary conditıens by the choice of the feedback controller in (2.5). We find that $k$ must solve the third orger partial differential equation given by

$$
\begin{aligned}
k_{x x x}+k_{y y y}+k_{y y}-k_{x x} & =-\lambda k, \\
k(x, 1) & =0, \\
k(x, x) & =0, \\
k_{x}(x, x) & =\frac{\lambda}{3}(1-x),
\end{aligned}
$$

where the PDE is considered on the triangular spatial domain

$$
T \equiv\left\{\left(x, y, \in \mathbb{R}^{2} \wedge x \in[0,1], y \in[x, 1]\right\} \text { (see Figure } 1\right. \text { below). }
$$

In order to prove the existence of a solution to the problem (2.8), we transform it into an integral equation and use the method of successive approximations. To this end, we first apply a change of variables with new variables $t \equiv y-x, s \equiv x+y$, and define the function $G(s, t) \equiv k(x, y)$. We find that $G$ satisfies the boundary value problem given by

$$
\begin{aligned}
2 G_{s s s}+6 G_{s t t}+4 G_{s t} & =-\lambda G, \\
G(s, 2-s) & =0 \\
G(s, 0) & =0 \\
G_{t}(s, 0) & =-\frac{\lambda}{6}(2-s)
\end{aligned}
$$

en the triangular domain

$$
T_{0} \equiv\{(s, t) \mid t \in[0,1], s \in[t, 2-t]\} \text { (see Figure } 2 \text { below) } .
$$


Integrating (2.9) by using the given boundary conditions, we obtain

$$
G(s, t)=-\frac{\lambda}{6} t(2-t-s)+\frac{1}{6} \int_{s}^{2-t} \int_{0}^{t} \int_{0}^{\tau}\left\{2 G_{s s s}+4 G_{s t}+\lambda G\right\}(\eta, \xi) d \xi d \tau d \eta .
$$

We solve (2.10) by using the method of successive approximations starting by setting

$$
G^{1}(s, t) \equiv-\frac{\lambda}{6} t(2-t-s)=\frac{\lambda}{6}\left[t^{2}+s t-2 t\right]
$$

and then defining

$$
G^{n+1}(s, t) \equiv G^{1}(s, t)+\frac{1}{6} \int_{s}^{2-t} \int_{0}^{t} \int_{0}^{\tau}\left\{2 G_{s s s}^{n}+4 G_{s t}^{n}+\lambda G^{n}\right\}(\eta, \xi) d \xi d \tau d i r
$$

for $n \geq 1$. We have the following lemma.

Lemma 2.13. Let $G^{1}$ and $G^{n+1}$ be defined by (2.11) and (2.12). Then there exists a $C^{3}$ function $G$ such that $\lim _{n \rightarrow \infty} G^{n}=G$ (uniformly), and moreover, $G$ solves the rntegral equation (2.10) as well as the boundary value problem given in (2.9).

Proof. We will prove the desired result by showing that $G^{n}$ is Cauchy in $C\left(T_{0}\right)$. Let us first introduce some notation. Let $P$ be the linear differential eperator given by

$$
P \varphi=\frac{1}{3} \varphi_{s s s}+\frac{2}{3} \varphi_{s t}+\frac{\lambda}{6} \varphi
$$

for $\varphi=\varphi(s, t)$. Let us also define the linear integration operator below

$$
I[\varphi](s, t) \equiv \int_{s}^{2-t} \int_{0}^{t} \int_{0}^{-} \tau_{\varphi}(\eta, \xi) d \xi d \tau d \eta
$$

Set $H^{1} \equiv G^{1}$ and $H^{n+1} \equiv I\left[P H^{n}\right]$ for $n \geq 1$ Then, we have

$$
\begin{gathered}
G^{2} \equiv G^{1}+I\left[P H^{1}\right]=G^{1}+H^{2}, \\
\left.G^{3}=G^{1}+I\left[P G^{2}\right]=G^{1}+I P G^{1}-P H^{2}\right]=G^{1}+I\left[P H^{1}\right]+H^{3}
\end{gathered}
$$$$
=G^{2}+H^{3} \text {. }
$$

More generally, $G^{n+1}=G^{n}+H^{n+1}$. Then, for $m>n$

$$
\max _{T_{0}}\left|G^{m}-G^{n}\right|=\max _{T_{0}}\left|\sum_{k=n+1}^{m}\left(G^{k}-G^{k-1}\right)\right| \leq \sum_{k=n+1}^{m} \max _{T_{0}}\left|G^{k}-G^{k-1}\right|=\sum_{k=n+1}^{m} \max _{T_{0}}\left|H^{k}\right| .
$$

From the above inecuality, it follows that in order to prove the Cauchy criteria for $G^{n}$, it is enough to prove that the series $\sum_{k=1}^{\infty} H^{k}$ is absolutely convergent. Proving the latter requires ontaining a good estimate on $\left|H^{k}\right|$.

Let us observe the first few $H^{k}$ 's to catch a pattern. For $k=1$, we have

$$
H^{1}=G^{1}=-\left(\frac{\lambda}{6}\right) t[(2-t)-s] .
$$

Then,

$$
P H^{1}=-\left(\frac{\lambda}{6}\right)^{2} t[(2-t)-s]+\left(\frac{\lambda}{6}\right)\left(\frac{2}{3}\right)
$$


BOOSTING THE DECAY OF SOLUTIONS OF THE LINEARIZED KORTEWEG-DE VRIES-BURGERS EQUATION TO For $k=2$, we have

$$
\begin{aligned}
& H^{2}=I\left[P H^{1}\right]=-\left(\frac{\lambda}{6}\right)^{2}\left[\frac{2 t^{3}}{3 \cdot 2}[(2-t)-s]\right. \\
& \left.-\frac{t^{4}}{4 \cdot 3}[(2-t)-s]-\frac{t^{3}}{3 \cdot 2} \frac{\left[(2-t)^{2}-s^{2}\right]}{2}\right]+\left(\frac{\lambda}{6}\right)\left(\frac{2}{3}\right) \frac{t^{2}}{2 \cdot 1}[(2-t)-s] \\
& =[(2-t)-s]\left[\left(\frac{\lambda}{6}\right)^{2} \frac{t^{4}}{4 \cdot 3}-\left(\frac{\lambda}{6}\right)^{2} \frac{2 t^{3}}{3 \cdot 2}+\left(\frac{\lambda}{6}\right)\left(\frac{2}{3}\right) \frac{t^{2}}{2 \cdot 1}\right] \\
& +\frac{1}{2}\left[(2-t)^{2}-s^{2}\right]\left[\left(\frac{\lambda}{6}\right)^{2}-2\right] .
\end{aligned}
$$

Then,

$$
\begin{aligned}
\text { (2.17) } P H^{2}=[(2-t)-s] & {\left[\left(\frac{\lambda}{6}\right)^{3} \frac{t^{4}}{4 \cdot 3}-\left(\frac{\lambda}{6}\right)^{3} \frac{2 t^{3}}{3 \cdot 2}+\left(\frac{\lambda}{6}\right)^{2}\left(\frac{2}{3}\right)\right.} \\
& +\frac{1}{2}\left[(2-t)^{2}-s^{2}\right]\left[\left(\frac{\lambda}{6}\right)^{3} t^{3}\right. \\
- & {\left[\left(\frac{\lambda}{6}\right)^{2}\left(\frac{2}{3}\right) \frac{4 \cdot 2}{4 \cdot 3}-\left(\frac{\lambda}{6}\right)^{2}\left(\frac{2}{3}\right) \frac{2 \cdot 3 t^{2}}{3 \cdot 2}+\left(\frac{\lambda}{6}\right)\left(\frac{2}{3} \frac{2 t}{2 \cdot 1}\right]-\frac{1}{2} 2 s\left[\left(\frac{\lambda}{6}\right)^{2}\left(\frac{2}{3}\right) \frac{3 t^{2}}{3 \cdot 2}\right] .\right.}
\end{aligned}
$$

For $k=3$, we have

(2.18) $H^{3}=I\left[P H^{2}\right]$

$$
\begin{aligned}
= & {[(2-t)-s]\left[\left(\frac{\lambda}{6}\right)^{3} \frac{2 t^{6}}{6 \cdot 5 \cdot 4 \cdot 3}-\left(\frac{\lambda}{6}\right)^{3} \frac{2^{2} t^{5}}{5 \cdot 4 \cdot 3 \cdot 2}+\left(\frac{\lambda}{6}\right)^{2}\left(\frac{2}{3}\right) \frac{2 t^{4}}{4 \cdot 3 \cdot 2 \cdot 1}\right] } \\
& \left.+[(2-t)-s]\left[-\frac{\lambda}{6}\right) \frac{t^{7}}{7 \cdot 6 \cdot 4 \cdot 3}+\left(\frac{\lambda}{6}\right)^{3} \frac{2 t^{6}}{6 \cdot 5 \cdot 3 \cdot 2}-\left(\frac{\lambda}{6}\right)^{2}\left(\frac{2}{3}\right) \frac{t^{5}}{5 \cdot 4 \cdot 2 \cdot 1}\right]
\end{aligned}
$$

$$
+\frac{1}{2}\left[\left(2-t^{2}-3^{2}\right]\left[-\left(\frac{\lambda}{6}\right)^{3} \frac{t^{6}}{6 \cdot 5 \cdot 4 \cdot 3}+\left(\frac{\lambda}{6}\right)^{3} \frac{2 t^{5}}{5 \cdot 4 \cdot 3 \cdot 2}-\left(\frac{\lambda}{6}\right)^{2}\left(\frac{2}{3}\right) \frac{2 t^{4}}{4 \cdot 3 \cdot 2 \cdot 1}\right]\right.
$$

$$
+[(2-t)-s] \frac{1}{2}\left[\left(\frac{\lambda}{6}\right)^{3} \frac{2^{2} t^{5}}{5 \cdot 4 \cdot 3 \cdot 2}\right]
$$




$$
\begin{aligned}
2.20)+[(2-t)-s] \frac{1}{2} & \left.-\left(\frac{\lambda}{6}\right)^{3} \frac{4 t^{6}}{6 \cdot 5 \cdot 3 \cdot 2}\right]+[(2-t)-s] \frac{1}{2}\left[\left(\frac{\lambda}{6}\right)^{3} \frac{t^{7}}{7 \cdot 6 \cdot 3 \cdot 2}\right] \\
& +\frac{1}{2 \cdot 3}\left[(2-t)^{3}-s^{3}\right]\left[\left(\frac{\lambda}{6}\right)^{3} \frac{t^{5}}{5 \cdot 4 \cdot 3 \cdot 2}\right] \\
-[(2-t)-s]\left[\left(\frac{\lambda}{6}\right)^{2}\left(\frac{2}{3}\right) \frac{4 \cdot t^{5}}{4 \cdot 3 \cdot 4 \cdot 5}-\left(\frac{\lambda}{6}\right)^{2}\left(\frac{2}{3}\right) \frac{2 \cdot 3 t^{4}}{3 \cdot 2 \cdot 3 \cdot 4}+\left(\frac{\lambda}{6}\right)\left(\frac{2}{3}\right)^{2} \frac{2 t^{3}}{2 \cdot 1 \cdot 2 \cdot 3}\right] & \frac{1}{2}\left[(2-t)^{2}-s^{2}\right]\left[\left(\frac{\lambda}{6}\right)^{2}\left(\frac{2}{3}\right) \frac{3 t^{4}}{3 \cdot 4 \cdot 3 \cdot 2}\right] \\
= & {[(2-t)-s]\left[-\left(\frac{\lambda}{6}\right)^{3} \frac{t^{7}}{7 \cdot 6 \cdot 4 \cdot 3}+2 \frac{1}{2}\right.}
\end{aligned}
$$

$$
\begin{aligned}
-\left(\frac{\lambda}{6}\right)^{3} \frac{2^{2} t^{5}}{5 \cdot 4 \cdot 3 \cdot 2}- & \left(\frac{\lambda}{6}\right)^{2}\left(\frac{2}{3}\right) \frac{t^{5}}{5 \cdot 4 \cdot 2 \cdot 1}+\frac{1}{2}\left(\frac{\lambda}{6}\right)^{3}-\frac{2^{2} t^{5}}{5 \cdot 4 \cdot 3 \cdot 2}-\left(\frac{\lambda}{6}\right)^{2}\left(\frac{2}{3}\right) \frac{4 \cdot t^{5}}{4 \cdot 3 \cdot 4 \cdot 5} \\
& \left(\frac{\lambda}{6}\right)^{2}\left(\frac{2}{3}\right) \frac{2 t^{4}}{4 \cdot 3 \cdot 2 \cdot 1}+\left(\frac{\lambda}{6}\right)\left(\frac{2}{3}\right) \frac{2 \cdot 3 t^{4}}{3 \cdot 2 \cdot 3 \cdot 4}
\end{aligned}
$$$$
\left.+\left(\frac{\lambda}{6}\right)\left(\frac{2}{3}\right)^{2} \frac{2 t^{3}}{2 \cdot \frac{1}{1} \cdot 2 \cdot 3}\right]
$$$$
+\frac{1}{2}\left[(2-t)^{2}-s^{2}\right]\left[-\left(\frac{\lambda}{6}\right)^{3} \frac{t^{6}}{6 \cdot 5 \cdot 4 \cdot 3}+\left(\frac{\lambda}{6}\right)^{3} \frac{2 t^{5}}{5 \cdot 4 \cdot 3 \cdot 2}\right.
$$$$
\left.-\left(\frac{\lambda}{6}\right)^{2}\left(\frac{2}{3}\right)-\frac{2 t^{4}}{4 \cdot 3 \cdot 2 \cdot 1}-\left(\frac{\lambda}{6}\right)^{2}\left(\frac{2}{3}\right) \frac{3 t^{4}}{3 \cdot 4 \cdot 3 \cdot 2}\right]
$$$$
+\frac{1}{2 \cdot 3}\left[(2-t)^{3}-s^{3}\right]\left[\left(\frac{\lambda}{6}\right)^{3} \frac{t^{5}}{5 \cdot 4 \cdot 3 \cdot 2}\right] \text {. }
$$

We intentionally did not perform the cancellations or simplifications in the above calculations to see the pattern more clearly. Indeed, we observe that $H^{k}$ has the following structure:

$$
H^{k}=\sum_{i=1}^{k} \frac{1}{i !}\left[(2-t)^{i}-s^{i}\right] \cdot\left[c_{3 k-1-i, i}^{k} t^{3 k-1-i}+c_{3 k-2-i, i}^{k} t^{3 k-2-i}+\ldots+c_{k-1+i, i}^{k} t^{k-1+i}\right]
$$

An important observation is that when we calculate $I\left[P H^{k-1}\right]$, the maximum number of terms of type $\left[(2-t)^{i}-s^{i}\right] t^{j}$ formed in $H^{k}$ is $3 k-5(k \geq 2)$ and the lowest denominator in the terms $c_{j, i}^{k}$ is $k$ ! (happens when $i=1$ ). Therefore, we can control the coefficient $3 k-5$ coming from the previous step of succession by cancelling it with $k$ in the $k$ ! and observing that $\frac{3 k-5}{k}<3$. In $k$ steps, this would give us a bound in the form $3^{k}$. Taking into 
consideration other terms, we estimate

$$
\left|c_{j, i}^{k}\right| \leq \frac{3^{k} 2^{k-i} \alpha^{k}}{(k-2+i) !}
$$

for $j \in\{k-1+i, k+i, \ldots, 3 k-1-i\}$ with $\alpha \equiv \max \left\{\frac{\lambda}{6}, \frac{2}{3}\right\}$. Note that the bound $2 k-1$ above comes from the fact that the maximum number of terms at the right hand side of $(2.22)$ is $3 k-1-1-(k-1+1)+1=2 k-1$ (this happens when $i=1)$. It follows that

$$
\max _{T_{0}}\left|H^{k}\right| \leq \frac{2^{k-1} \alpha^{k} 3^{k}(2 k-1)}{(k-1) !}
$$

Now, it is easy to show that

$$
\sum_{k=1}^{\infty} \max _{T_{0}}\left|H^{k}\right|<\infty
$$

Indeed,

$$
\sum_{k=1}^{\infty} \frac{2^{k-1} \alpha^{k} 3^{k}(2 k-1)}{(k-1) !} \leq 6 \alpha \sum_{k=1}^{\infty} \frac{(12 \alpha)^{k-1}}{(k-1) !}<6 \alpha e^{12 \alpha}<\infty
$$

Remark 2.24. We also calculated the bounds on the first few $H^{n}$ 's numerically with Wolfram Mathematica ${ }^{\circledR} 11$ ) (for $\lambda=1$ ) and found the values given in Table 1 . These numerical simulations show that the convergence of the successive approximations is in fact quite fast and roughly at the order of $10^{-2 n+3}$.

Now switching back to old variables $x, y$ via $k(x, y) \equiv G(x+y, y-x)$ we obtain the existence of the kernel function $k$. Therefore the existence of the boundary controller is proven. We can now conclude that $w$ decays in the mean square sense. Figure 3 shows that the control effort increases if one desires to stabilize the system more quickly.

The decay of $w$ does not immediately tell us that $u$ also decays. In order to prove this, we need to obtain the invers of the relation between $L^{2}(\Omega)$-norms of $u$ and $w$ given in Remark 2.6. To this end, we define an inverse transformation

$$
u(x, t) \equiv w(x, t)+\int_{x}^{1} l(x, y) w(y, t) d y
$$

where $l$ is a continuous kernel function to be found. Similar to the proof of the existence of $k$, one can obtain a third order PDE which can be solved for $\ell$ in the triangular domain $T$ :

$$
\left\{\begin{array}{l}
\ell_{x x x}+\ell_{y y y}+\ell_{y y}-\ell_{x x}=\lambda \ell, \\
\ell(x, 1)=0 \\
\ell(x, x)=0 \\
\ell_{x}(x, x)=\frac{\lambda}{3}(1-x) .
\end{array}\right.
$$

The above PDE can be transformed into another third order PDE, which is equivalent to an integral equation whose solution is obtained via successive approximations in the same way we treated (2.8). See Figure 4 for a graph of the inverse control gain $l(0, y)$. 
It is important to notice that, from (2.25), we have the estimate

$$
\|u(t)\|_{L^{2}(\Omega)} \lesssim\|w(t)\|_{L^{2}(\Omega)}
$$

which gives us an exponential decay rate for $u$, since $w$ satisfies (2.3). In (2.27), the constant of the inequality depends of course on the kernel function $\ell$. We conclude this section with the following proposition, whose proof follows from the analysis given above.

Proposition 2.28. Let $\lambda>0$. Then, there exists a kernel function $k=k(x, y)$ such that the solution of the linearized KdV-Burgers equation in (1.6) subject to the boundary controller $U(t)=\int_{0}^{1} k(0, y) u(y, t) d y$ satisfies

$$
\|u(t)\|_{L^{2}(\Omega)} \lesssim e^{-\lambda t}\left\|u_{0}\right\|_{L^{2}(\Omega)}
$$

for $t \geq 0$.

2.2. Nonlinear Stability. In this section, we consider the nonlinear model (1 1) with the feedback controller constructed in the previous section. By using the rransformation given in (2.4), we obtain the following PDE from (1.1), noting that $\ell(x, x)=-\mathrm{e}$ :

$$
\begin{aligned}
w_{t}(x, t)- & w_{x x}(x, t)+w_{x x x}(x, t)+\lambda w(x, t) \\
& \left.=-\left(w(x, t)+\int_{x}^{1} \ell(x, y) w(y, t) d y\right)\left(v_{x}(x, t)\right)+\int_{x}^{1} \ell_{x}(x, y) w(y, t) d y\right)
\end{aligned}
$$

with homogeneous boundary conditions

$$
w(0, t)=0, w(1, t)=0 \text {, and } \omega_{x}(1, t)=0 .
$$

Multiplying (2.30) by $w(x, t)$ and integrating over $(5,1)$, we obtain

$$
\begin{aligned}
& \int_{0}^{1} w(x, t) w_{t}(x, t) d x=\int_{0}^{1} w(x, t) w_{x x}(x, t) d x-\int_{0}^{1} w(x, t) w_{x x x}(x, t) d x \\
& -\lambda \int_{0}^{1} w^{2}(x, t) d x-\int_{0}^{1} y^{2}(x, t) w_{x}(x, t) d x-\int_{0}^{1} w^{2}(x, t)\left[\int_{x}^{1} \ell_{x}(x, y) w(y, t) d y\right] d x \\
& -\int_{0}^{1} w(x, t)\left[\int_{x}^{1} \ell(x, y) w(y, t) d y\right]\left[\int_{x}^{1} \ell_{x}(x, y) w(y, t) d y\right] d x
\end{aligned}
$$

Using integration-by parts and Cauchy-Schwarz inequality, we obtain

$$
\leq-\left\|w_{x}(t)\right\|_{L^{2}(\Omega)}^{2}-\frac{1}{2}\left|w_{x}(0, t)\right|^{2}+\left(\frac{3}{2}\|\ell\|_{C^{1}(T)}+\|\ell\|_{C^{1}(T)}^{2}\right)\|w(t)\|_{L^{2}(\Omega)}^{3} .
$$

Hence, we have the following inequality:

$$
y^{\prime}+2 \lambda y-c y^{\frac{3}{2}} \leq 0
$$


where $y(t) \equiv\|w(t)\|_{L^{2}(\Omega)}^{2}$ and $c=2\left(\frac{3}{2}\|\ell\|_{C^{1}(T)}+\|\ell\|_{C^{1}(T)}^{2}\right)$. Solving the inequality (2.34) and assuming $\left\|w_{0}\right\|_{L^{2}(\Omega)}<\frac{\lambda}{c}$, we get

$$
\|w(t)\|_{L^{2}(\Omega)}^{2}=y(t) \leq \frac{1}{\left[\left(\frac{1}{\left\|w_{0}\right\|_{L^{2}(\Omega)}}-\frac{c}{2 \lambda}\right) e^{\lambda t}+\frac{c}{2 \lambda}\right]^{2}}<\frac{1}{\left[\frac{e^{\lambda t}}{2\left\|w_{0}\right\|_{L^{2}(\Omega)}}\right]^{2}} .
$$

Recall that $\left\|w_{0}\right\|_{L^{2}(\Omega)} \lesssim\left\|u_{0}\right\|_{L^{2}(\Omega)}$ (see Remark 2.6). Combining this with (2.27) and (2.35), we deduce

$$
\|u(t)\|_{L^{2}(\Omega)} \lesssim\left\|u_{0}\right\|_{L^{2}(\Omega)} e^{-\lambda t}, \text { for } t \geq 0 .
$$

Hence, the proof of Theorem 1.3 is complete.

\section{OBSERVER DESIGN}

3.1. Linearized equation. In this section, we consider the linearize $\mathrm{Kd} \mathrm{V}$ - Burgers model with boundary actuation given in (1.6). We suppose that the measurements for the solution are not available across the domain and there is a sensor only at the right end point of the domain, which measures the quantity $y(t)=\partial_{x}^{2} u(1, t)$. To this end. we consider the observer given in (1.7).

Our goal is to find a function $p_{1}=p_{1}(x)$ such that $u-u$ goes to zero as $t \rightarrow \infty$ in a physcially appropriate sense. In order to achieve this, we define the error function $\tilde{u} \equiv u-\hat{u}$. Then, $\tilde{u}$ satisfies

$$
\left\{\begin{array}{l}
\tilde{u}_{t}-\tilde{u}_{x x}+\tilde{u}_{x x x}-p_{1}(x) \tilde{u}_{x x}(1, t)=0 \\
\tilde{u}(0, t)=\tilde{u}(1, t)=\tilde{u}_{x}(1, t)=0 \\
\tilde{u}(x, 0)=u_{0}-\tilde{u}_{0} .
\end{array}\right.
$$

The next step is to find a kernel function $p=p(x, y)$ such that the transformation

$$
\tilde{u}(x)=\tilde{w}(x)-\int_{x}^{1} p(x, y) \tilde{w}(y, t) d y
$$

makes the error system (3.1) equivalent to the following exponentially stable linear $\mathrm{KdV}$ Burgers equation:

$$
\left\{\begin{array}{l}
\tilde{w}_{t}-\tilde{w}_{x x}+\tilde{w}_{x x x}+\lambda \tilde{w}=0 \\
\tilde{w}(0, t)=\tilde{w}(1, t)=\tilde{w}_{x}(1, t)=0 .
\end{array}\right.
$$

Calculating temorat and spatial derivatives of $\tilde{u}$ which is given by (3.2), substituting these into (3.1), and using the boundary conditions in (3.1), we find out that $p_{1}(x)$ should be chosen to be $p(x, 1)$, where the kernel function $p$ solves the following PDE posed in the triangular domain $T$ :

$$
\left\{\begin{array}{l}
p_{x x x}+p_{y y y}+p_{y y}-p_{x x}=\lambda p \\
p(x, x)=0 \\
p_{x}(x, x)=\frac{\lambda}{3} x \\
p(0, y)=0 .
\end{array}\right.
$$

The above model can be solved similarly to (2.8) by a change variables given by $x^{\prime} \equiv 1-y$ and $y^{\prime} \equiv 1-x$. Note that the signs of lower order derivatives (second order derivatives) will be reversed with such a change of variables. This constrasts with KdV since first order derivatives are used there. See Figure 5 for the graph of the control gain $p_{1}(x)$. 
The transformation given in (3.2) is invertible in the sense that there exists a kernel function $r(x, y)$ such that

$$
\tilde{w}(x)=\tilde{u}(x)+\int_{x}^{1} r(x, y) \tilde{u}(y, t) d y
$$

and $r$ satisfies

$$
\left\{\begin{array}{l}
r_{x x x}+r_{y y y}+r_{y y}-r_{x x}=-\lambda r \\
r(x, x)=0 \\
r_{x}(x, x)=\frac{\lambda}{3} x \\
r(0, y)=0
\end{array}\right.
$$

We consider the back-stepping transformation as well as its inverse given before now for $\hat{u}$. That is,

$$
\hat{w}(x)=\hat{u}(x)-\int_{x}^{1} k(x, y) \hat{u}(y, t) d y
$$

and

$$
\hat{u}(x)=\hat{w}(x)+\int_{x}^{1} \ell(x, y) \hat{w}(y, t) d y,
$$

where $k$ and $\ell$ satisfy (2.8) and (2.26), respectively. The transformation (3.7) gives the following equation in $\hat{w}$ :

$$
\left\{\begin{array}{l}
\hat{w}_{t}-\hat{w}_{x x}+\hat{w}_{x x x}+\lambda \hat{w}=-\left\{p_{1}(x)-\gamma_{c}^{1} k(x, y) p_{1}(y) d y\right\} \tilde{w}_{x x}(1, t), \\
\hat{w}(0, t)=\hat{w}(1, t)=\hat{w}_{x}(1, t)=0
\end{array}\right.
$$

Lemma 3.10. Let $\tilde{w}$ be a solution of (3.3). Then,

$$
\mid \tilde{w}_{x x}(1, t) \leqslant\left(\|\tilde{w}(t)\|_{L^{2}(\Omega)}+\left\|\tilde{w}_{t}(t)\right\|_{L^{2}(\Omega)}\right) \text {. }
$$

Proof. We multiply (3.3) with $x \tilde{w}_{x x}$ and integrate over $\Omega=(0,1)$. We obtain

$$
\int_{0}^{1} x \tilde{w}_{t} \tilde{w}_{x x} d x-\int_{0}^{1} x \tilde{w}_{x x}^{2} d x+\frac{1}{2}\left|\tilde{w}_{x x}(1, t)\right|^{2}-\frac{1}{2} \int_{0}^{1} \tilde{w}_{x x}^{2} d x-\lambda \int_{0}^{1} x \tilde{w}_{x}^{2} d x=0 .
$$

Using the Cauchy Schwarz inequality on the first term above, we estimate

$$
\left\langle\left.\tilde{w}_{x x}(1, t)\right|^{2} \leq\left\|\tilde{w}_{t}\right\|_{L^{2}(\Omega)}^{2}+3\left\|\tilde{w}_{x x}\right\|_{L^{2}(\Omega)}^{2}+\lambda\|\tilde{w}\|_{L^{2}(\Omega)}^{2} .\right.
$$

From the main equation and the triangle inequality, we have

$$
\left\|\tilde{w}_{x x x}\right\|_{L^{2}(\Omega)}^{2} \leq 3\left(\lambda^{2}\|\tilde{w}\|_{L^{2}(\Omega)}^{2}+\left\|\tilde{w}_{x x}\right\|_{L^{2}(\Omega)}^{2}+\left\|\tilde{w}_{t}\right\|_{L^{2}(\Omega)}^{2}\right) .
$$

Recall that we have the Gagliardo-Nirenberg inequality

$$
\left\|\tilde{w}_{x x}\right\|_{L^{2}(\Omega)} \leq\left\|\tilde{w}_{x x x}\right\|_{L^{2}(\Omega)}^{\frac{2}{3}}\|\tilde{w}\|_{L^{2}(\Omega)}^{\frac{1}{3}} .
$$

Taking squares of both sides of (3.14) and applying $\epsilon$-Young's inequality, we obtain

$$
\left\|\tilde{w}_{x x}\right\|_{L^{2}(\Omega)}^{2} \leq \epsilon\left\|\tilde{w}_{x x x}\right\|_{L^{2}(\Omega)}^{2}+\frac{1}{\epsilon}\|\tilde{w}\|_{L^{2}(\Omega)}^{2}
$$


for $\epsilon>0$ small enough and fixed. Using this in (3.13), we get

$$
\left\|\tilde{w}_{x x x}\right\|_{L^{2}(\Omega)}^{2} \leq \frac{3\left(1+\lambda^{2}+\frac{3}{\epsilon}\right)}{1-3 \epsilon}\left(\|\tilde{w}\|_{L^{2}(\Omega)}^{2}+\left\|\tilde{w}_{t}\right\|_{L^{2}(\Omega)}^{2}\right)
$$

which allows to write

$$
\left\|\tilde{w}_{x x}\right\|_{L^{2}(\Omega)}^{2} \leq\left[\frac{\epsilon\left(1+\lambda^{2}+\frac{3}{\epsilon}\right)}{1-3 \epsilon}+\frac{1}{\epsilon}\right]\left(\|\tilde{w}\|_{L^{2}(\Omega)}^{2}+\left\|\tilde{w}_{t}\right\|_{L^{2}(\Omega)}^{2}\right) .
$$

Hence, (3.12) can be rewritten in the form:

$$
\left|\tilde{w}_{x x}(1, t)\right|^{2} \lesssim\|\tilde{w}\|_{L^{2}(\Omega)}^{2}+\left\|\tilde{w}_{t}\right\|_{L^{2}(\Omega)}^{2}
$$

and the proof of the lemma is complete.

Now, we define an energy functional for the above system by

$$
E(t) \equiv\|\hat{w}(t)\|_{L^{2}(\Omega)}^{2}+\|\tilde{w}(t)\|_{L^{2}(\Omega)}^{2}+\left\|\tilde{w}_{t}(t)\right\|_{L^{2}(\Omega)}^{2}
$$

Multiplying (3.9) by $\hat{w}$ and integrating over $\Omega$, we obtain

$$
\frac{1}{2} \frac{d}{d t}\|\hat{w}(t)\|_{L^{2}(\Omega)}^{2}+\left\|\hat{w}_{x}(t)\right\|_{L^{2}(\Omega)}^{2}+\frac{1}{2}\left|\hat{w}_{x}(0, t)\right|^{2}+\lambda \| \hat{w}\left(t \|_{L^{2}(\Omega)}=\tilde{w}_{x x}(1, t) \int_{0}^{1} \Psi \hat{w} d x,\right.
$$

where $\Psi(x)=-\left\{p_{1}(x)-\int_{x}^{1} k(x, y) p_{1}(y) d y\right\}$. Combining the above identitity with Lemma 3.10 and using $\epsilon$-Young's inequality, we get

$$
\frac{d}{d t}\|\hat{w}(t)\|_{L^{2}(\Omega)}^{2} \leq-(2 \lambda-\epsilon)\|\hat{w}(t)\|_{L^{2}\left(\Omega_{2}\right)}^{2}+C_{\epsilon}\left(\|\tilde{w}\|_{L^{2}(\Omega)}^{2}+\left\|\tilde{w}_{t}\right\|_{L^{2}(\Omega)}^{2}\right),
$$

where $C_{\epsilon}$ depends on $\epsilon, \lambda$, and $\|\Psi\|_{\infty}$ As we have shown in (2.2), $\tilde{w}$ can be also shown to satisfy

which is equivalent to writing

$$
\frac{d}{d t}\|\tilde{w}(t)\|_{L^{2}(\Omega)}^{2} \leq-2 \lambda\|\tilde{w}(t)\|_{L^{2}(\Omega)}^{2}, t \geq 0
$$

$$
\|\hat{\nu}(t)\|_{L^{2}(\Omega)}^{2} \leq\left\|\tilde{w}_{0}\right\|_{L^{2}(\Omega)}^{2} e^{-2 \lambda t .}
$$

Now, we differentiate (3.3) in the variable $t$, then multiply both sides by $\tilde{w}_{t}$ and integrate over $\Omega$. Therefore, we have

$$
\frac{1 d}{2 d t} \tilde{w}_{t}(t)\left\|_{L^{2}(\Omega)}^{2}=-\right\| \tilde{w}_{t x}(t)\left\|_{L^{2}(\Omega)}^{2}-\frac{1}{2}\left|\bar{w}_{t x}(0)\right|^{2}-\lambda\right\| \tilde{w}_{t}(t) \|_{L^{2}(\Omega)}^{2} .
$$

It follows that

(3.24) $\left\|\tilde{w}_{t},(\lambda)\right\|_{J^{2}(\Omega)} \leq\left\|\tilde{w}_{t}(0)\right\|_{L^{2}(\Omega)} e^{-\lambda t}=\left\|\tilde{w}_{0}^{\prime \prime}-\tilde{w}_{0}^{\prime \prime \prime}-\lambda \tilde{w}_{0}\right\|_{L^{2}(\Omega)} e^{-\lambda t} \lesssim\left\|\tilde{w}_{0}\right\|_{H^{3}(\Omega)} e^{-\lambda t}, t \geq 0$.

Combining (3.21)-(3.24), we deduce

$$
E^{\prime}(t) \leq-(2 \lambda-\epsilon) E(t)+C_{\epsilon, w_{0}} e^{-2 \lambda t},
$$

where $C_{\epsilon, w_{0}}>0$ is a constant that depends on $\epsilon,\left\|\tilde{w}_{0}\right\|_{L^{2}(\Omega)},\left\|\tilde{w}_{0}^{\prime \prime}-\tilde{w}_{0}^{\prime \prime \prime}-\lambda \tilde{w}_{0}\right\|_{L^{2}(\Omega)}, \lambda$, and $\|\Psi\|_{\infty}$. Multiplying both sides by $e^{(2 \lambda-\epsilon) t}$ and integrating, we obtain

$$
E(t) \leq C_{\epsilon, w_{0}} e^{-(2 \lambda-\epsilon) t}, t \geq 0 .
$$

Based on the above analysis, we can now prove Theorem 1.8. 
Proof of Theorem 1.8. Pick some $\tilde{\lambda}>\lambda$, e.g., $\tilde{\lambda} \equiv \lambda+\frac{\epsilon}{2}$. We can do the entire analysis above starting with $\tilde{\lambda}$ rather than $\lambda$ and obtain $E(t) \leq C_{\epsilon, w_{0}} e^{-(2 \tilde{\lambda}-\epsilon) t} \leq C_{\epsilon, w_{0}} e^{-2 \lambda t}$ where in this case the constant $C_{\epsilon, w_{0}}$ as well as all kernel functions depend on $\tilde{\lambda}$. We observe from (3.2) that $\|\tilde{u}(t)\|_{H^{3}(\Omega)} \lesssim\|\tilde{w}(t)\|_{H^{3}(\Omega)}$. Similarly, it follows from (3.8) that $\|\hat{u}(t)\|_{L^{2}(\Omega)} \lesssim\|\hat{w}(t)\|_{L^{2}(\Omega)}$. In addition, we can find a similar relation between the initial data using the invertibility of the back-stepping transformations, i.e., $\left\|\tilde{w}_{0}\right\|_{H^{3}(\Omega)} \lesssim\left\|u_{0}-\hat{u}_{0}\right\|_{H^{3}(\Omega)}$ and $\left\|\hat{w}_{0}\right\|_{L^{2}(\Omega)} \lesssim\left\|\hat{u}_{0}\right\|_{L^{2}(\Omega)}$. Combining the above arguments, we conclude that

$$
\|u-\hat{u}\|_{H^{3}(\Omega)}+\|\hat{u}\|_{L^{2}(\Omega)} \lesssim\left(\left\|u_{0}-\hat{u}_{0}\right\|_{H^{3}(\Omega)}+\left\|\hat{u}_{0}\right\|_{L^{2}(\Omega)}\right) e^{-\lambda t} .
$$

3.2. Other boundary conditions. Consider the linearized KdVB equation in (1.10\%. If one could fully observe the system (1.10), then we could stabilize the systen with the predetermined rate of decay by designing a boundary controller $U(t)=\int_{0}^{1} k(\pi, y) u(y, t) d y$, where $k$ solves

$$
\begin{aligned}
k_{x x x}+k_{y y y}+k_{y y}-k_{x x} & =-\lambda k \\
k_{y}(x, 1)+k_{y y}(x, 1) & =0 \\
k(x, x) & =0 \\
k_{x}(x, x) & =-\frac{1}{3}(1-r)
\end{aligned}
$$

on the triangular spatial domain $T$. However, here we will consider the observer in (1.11) assuming one can only get partial information (in this case it is $y(t)=u(1, t)$ ) about the system (1.10).

(3.27) can be solved by using the same procedure in Section 2.1. By using the change of variables $G(s, t) \equiv k(x, y)$ with $t \equiv y-x, s=x+y$, one gets the following PDE model:

$$
\begin{aligned}
2 G_{s s s}+\hat{\epsilon} G_{s t t}+4 G_{s t} & =-\lambda G, \\
G_{t t}+G_{s}+2 G_{s t}+G_{s}+\left.G_{t}\right|_{t=2-s} & =0, \\
G(s, 0) & =0, \\
G_{t}(s, 0) & =-\frac{\lambda}{6}(2-s),
\end{aligned}
$$

where $(s, t) \in T_{0}$.

After some computations, we obtain

$$
\begin{aligned}
G(s, t)=-\frac{\lambda}{6} t(2-s) & -\int_{0}^{t} 2\left(G_{s}(s, \tau)+G(s, \tau)\right) d \tau \\
& -\int_{0}^{t} \int_{0}^{\tau}\left(G_{s s}(s, \xi)+G_{s}(s, \xi)\right) d \xi d \tau \\
& -\frac{1}{6} \int_{0}^{t} \int_{0}^{\tau} \int_{s}^{2-\xi}\left(4 G_{s s s}+12 G_{s s t}+6 G_{s s}+2 G_{s t}-\lambda G\right)(\eta, \xi) d \eta d \xi d \tau .
\end{aligned}
$$

We solve the above equation again with iteration similar to what we have done in Lemma 2.12. The graph of the corresponding control gain is given in Figure 6 . 
We set the error $\tilde{u} \equiv u-\hat{u}$, which satisfies

$$
\left\{\begin{array}{l}
\tilde{u}_{t}-\tilde{u}_{x x}+\tilde{u}_{x x x}-p_{1}(x) \tilde{u}(1, t)=0 \\
\tilde{u}(0, t)=\tilde{u}_{x}(1, t)=\tilde{u}_{x x}(1, t)=0 \\
\tilde{u}(x, 0)=u_{0}-\hat{u}_{0}
\end{array}\right.
$$

We consider the transformation in (3.2), which transforms (3.30) into

$$
\left\{\begin{array}{l}
\tilde{w}_{t}-\tilde{w}_{x x}+\tilde{w}_{x x x}+\lambda \tilde{w}=0 \\
\tilde{w}(0, t)=\tilde{w}_{x}(1, t)=\tilde{w}_{x x}(1, t)=0 .
\end{array}\right.
$$

provided that $p_{1}(x)=p(x, 1)$ and $p$ solves

$$
\left\{\begin{array}{l}
p_{x x x}+p_{y y y}+p_{y y}-p_{x x}=\lambda p \\
p(x, x)=0 \\
p_{x}(x, x)=\frac{\lambda}{3}(x-1), \\
p(0, y)=0 .
\end{array}\right.
$$

By using the change of variables $G(s, t) \equiv k(x, y)$ with $t \equiv y-x, s=x+y$, one gets the following PDE model:

$$
\begin{aligned}
2 G_{s s s}+6 G_{s t t}+4 G_{s t} & =\lambda G, \\
G(s, s) & =0 \\
G(s, 0) & =0, \\
G_{t}(s, 0) & =-\frac{\lambda}{6}(2-s),
\end{aligned}
$$

where $(s, t) \in T_{0}$. After some calculations, we get

$$
G(s, t)=-\frac{\lambda}{6} t(t-s)+\int_{s}^{t} \int_{0}^{t} \int_{0}^{t}\left(\frac{1}{3} G_{s s s}+\frac{2}{3} G_{s t}-\frac{\lambda}{6} G\right)(\eta, \xi) d \xi d \tau d \eta .
$$

The above integral equation can be solved by iteration and the solution is as in Figure 7 .

The inverse transformatior is defined by (3.5) where in this case $r$ satisfies

$$
\left\{\begin{array}{l}
r_{x x y}+r_{y y y}+r_{y y}-r_{x x}=-\lambda r, \\
r(x, x)=0 \\
r_{x}(x, x)=\frac{\lambda}{3}(x-1), \\
r(0, y)=0 .
\end{array}\right.
$$

We also consider the same transformations given in (3.7) and (3.8). Then $\hat{w}$ satisfies

$$
\left\{\begin{array}{l}
\hat{w}_{t}-\hat{w}_{x x}+\hat{w}_{x x x}+\lambda \hat{w}=-\left\{p_{1}(x)-\int_{x}^{1} k(x, y) p_{1}(y) d y\right\} \tilde{w}(1, t), \\
\hat{w}(0, t)=\hat{w}_{x}(1, t)=\hat{w}_{x x}(1, t)=0 .
\end{array}\right.
$$

We a tinte the energy functional for the above system by

$$
E(t) \equiv\|\hat{w}(t)\|_{L^{2}(\Omega)}^{2}+\|\tilde{w}(t)\|_{L^{2}(\Omega)}^{2}
$$

and the modified energy functional given by

$$
E_{\rho}(t) \equiv\|\hat{w}(t)\|_{L^{2}(\Omega)}^{2}+\rho\|\tilde{w}(t)\|_{L^{2}(\Omega)}^{2}
$$


for $\rho>0$. It is clear that $\frac{\rho}{\rho+1} E \leq E_{\rho} \leq(1+\rho) E$. Therefore, $E$ and $E_{\rho}$ are asymptotically equivalent.

We now give a simple inequality by the following lemma.

Lemma 3.39. Let $\tilde{w}$ be a solution of (3.31). Then, $|\tilde{w}(1, t)| \lesssim\left\|\tilde{w}_{x}\right\|_{L^{2}(0,1)}$.

Proof. We first write $\tilde{w}^{2}(1, t)=\frac{1}{2} \int_{0}^{1}\left(\tilde{w}^{2}\right)_{x} d x$ by using the boundary condition $\tilde{w}(0, t)=0$. But the right hand side of this equality can be rewritten as $\int_{0}^{1} \check{w}_{x} d x$, which is bonruded by $\|\tilde{w}\|_{L^{2}(0,1)}\left\|\tilde{w}_{x}\right\|_{L^{2}(0,1)}$. On the other hand, by Poincaré inequality $\left.\|\tilde{w}\|_{L^{2}(0,1)} \lesssim \| \tilde{w} / x_{x} / L^{2}(0) 1\right)$. Hence, the result follows.

Multiplying (3.36) by $\hat{w}$ and integrating over $\Omega$, we obtain

$$
\frac{1}{2} \frac{d}{d t}\|\hat{w}(t)\|_{L^{2}(\Omega)}^{2}+\left\|\hat{w}_{x}(t)\right\|_{L^{2}(\Omega)}^{2}+\frac{1}{2}\left|\hat{w}_{x}(0, t)\right|^{2}+\lambda\|\hat{w}(t)\|_{L^{2}(\Omega)}^{2}=\tilde{w}(1, t) \hat{\Psi}^{1} \hat{w} d x
$$

where $\Psi(x)=-\left\{p_{1}(x)-\int_{x}^{1} k(x, y) p_{1}(y) d y\right\}$. Combining the above identitity with Lemma 3.39 and using $\epsilon$-Young's inequality, we get

$$
\frac{d}{d t}\|\hat{w}(t)\|_{L^{2}(\Omega)}^{2} \leq-(2 \lambda-\epsilon)\|\hat{w}(t)\|_{L^{2}(\Omega y}^{2}+c_{\epsilon}\left\|\tilde{w}_{x}(t)\right\|_{L^{2}(0,1)}^{2},
$$

where $C_{\epsilon}$ depends on $\epsilon$ and $\|\Psi\|_{\infty}$. $\tilde{w}$ can be easiliy shown to satisfy

$$
\frac{d}{d t}\|\tilde{w}(t)\|_{L^{2}(\Omega)}^{2}+2\left\|\tilde{w}_{x}(t)\right\|_{L^{2}(\Omega)}^{2} \leqslant-2 \lambda\|\tilde{v}(t)\|_{L^{2}(\Omega)}^{2}, t \geq 0
$$

Therefore, for $\rho$ sufficiently large and $\epsilon$ sufficiently small, we obtain $\dot{E}_{\rho}(t) \leq-(2 \lambda-\epsilon) E_{\rho}(t)$. The last inequality, gives the exponentrar decay oi the pair $(\hat{w}, \tilde{w})$ in $L^{2}$-sense with a rate almost equal to $\lambda$. Hence, we can now give a proof for Theorem 1.12.

Proof of Theorem 1.12. Pick some $\tilde{\lambda}>\lambda$. We can do the entire analysis above starting with $\tilde{\lambda}$ rather than $\lambda$ and obtain $E(t) \leq C_{\epsilon, \rho, w_{0}} e^{-(2 \tilde{\lambda}-\epsilon) t} \leq C_{\epsilon, \rho, w_{0}} e^{-2 \lambda t}$ where in this case the constant $C_{\epsilon, \rho, w_{0}}$ as well as all kernel functions depend on $\tilde{\lambda}$. We observe from (3.2) that $\|\tilde{u}(t)\|_{L^{2}(\Omega)} \lesssim\|\tilde{w}(t)\|_{L^{2}(\Omega)}$ Sinilarly, it follows from (3.8) that $\|\hat{u}(t)\|_{L^{2}(\Omega)} \lesssim\|\hat{w}(t)\|_{L^{2}(\Omega)}$. In addition, we can find a similar relation between the initial data using the invertibility of the back-stepping transformations, i.e., $\left\|\tilde{w}_{0}\right\|_{L^{2}(\Omega)} \lesssim\left\|u_{0}-\hat{u}_{0}\right\|_{L^{2}(\Omega)}$ and $\left\|\hat{w}_{0}\right\|_{L^{2}(\Omega)} \lesssim\left\|\hat{u}_{0}\right\|_{L^{2}(\Omega)}$. Combining the above arguments, we conclude that

$$
\|u-\hat{u}\|_{L^{2}(\Omega)}+\|\hat{u}\|_{L^{2}(\Omega)} \lesssim\left(\left\|u_{0}-\hat{u}_{0}\right\|_{L^{2}(\Omega)}+\left\|\hat{u}_{0}\right\|_{L^{2}(\Omega)}\right) e^{-\lambda t} .
$$

\section{REFERENCES}

Anick C. J., Bona, J. L., \& Schonbek, M. E. (1989). Decay of solutions of some nonlinear wave equations. J. Differential Equations, 81(1), 1-49. Available from http://dx.doi . org/10.1016/0022-0396(89) 90176-9

Balogh, A., \& Krstic, M. (2000). Boundary control of the Korteweg-de Vries-Burgers equation: further results on stabilization and well-posedness, with numerical demonstration. IEEE Trans. Automat. Control, 45(9), 1739-1745. Available from http:// dx.doi.org/10.1109/9.880639 
Bona, J. L., Sun, S. M., \& Zhang, B.-Y. (2003). A nonhomogeneous boundary-value problem for the Korteweg-de Vries equation posed on a finite domain. Comm. Partial Differential Equations, 28(7-8), 1391-1436. Available from http://dx.doi.org/10.1081/ PDE-120024373

Cavalcanti, M. M., Domingos Cavalcanti, V. N., Komornik, V., \& Rodrigues, J. H. (2014). Global well-posedness and exponential decay rates for a $\mathrm{KdV}$-Burgers equation with indefinite damping. Ann. Inst. H. Poincaré Anal. Non Linéaire, 31 (5), 1079-1190. Available from http://dx.doi.org/10.1016/j.anihpc.2013.08.003

Cerpa, E. (2007). Exact controllability of a nonlinear Korteweg-de Vries equation on a critical spatial domain. SIAM J. Control Optim., 46(3), 877-899. Available from http://dx.doi.org/10.1137/06065369X

Cerpa, E., \& Coron, J.-M. (2013). Rapid stabilization for a Korteweg-de Vries equation from the left Dirichlet boundary condition. IEEE Trans. Automat. Contral, 58(7), 1688-1695. Available from http://dx.doi.org/10.1109/TAC.2013.2211.179

Cerpa, E., \& Crépeau, E. (2009). Boundary controllability for the nonlinear Korteweg-de Vries equation on any critical domain. Ann. Inst. H. Poincaré Anal. Non Linéaire, 26(2), 457-475. Available from http://dx.doi.org/10.1015/j.anihpc.2007.11 .003

Hasan, A. (2016). Output-feedback stabilization of the Korteweg de-Vries equation. Mediterranean Conference on Control and Automation. Available from https://arxiv.org/ abs $/ 1603.08750$

Jia, C. (2016). Boundary feedback stabilization of the Korteweg-de Vries-Burgers equation posed on a finite interval. J. Math. Anai. Apnl., 444(1), 624-647. Available from http://dx.doi.org/10.1016/j.jmaa.2016 06.063

Jia, C., \& Zhang, B.-Y. (2012). Boundary stabilization of the Korteweg-de Vries equation and the Korteweg-de Vries-Burgers equation. Acta Appl. Math., 118, 25-47. Available from http://dx.doi.org/10.100\%/s10440-012-9676-4

Komornik, V., Russell, D. L., \& Zhang, B. Y. (1991). Stabilisation de l'équation de Kortewegde Vries. C. R. Acad. Sci. Paris Sér. I Math., 312(11), 841-843.

Krstic, M., \& Smyshlyaev. A. (2058). Boundary control of PDEs (Vol. 16). Society for Industrial and Applied Mathematics (SIAM), Philadelphia, PA. Available from http://dx.do\%. org/10.1137/1.9780898718607 (A course on backstepping designs)

Laurent, C., Rosier. L., \& Zhang, B.-Y. (2010). Control and stabilization of the Korteweg-de Vries equation on a periodic domain. Comm. Partial Differential Equations, 35(4), 707-74.4. Available from http://dx.doi.org/10.1080/03605300903585336

Linares, F. \& Pazoto, A. F. (2007). On the exponential decay of the critical generalized Korteweg-de Vries equation with localized damping. Proc. Amer. Math. Soc., 135(5), 1515-1522. Available from http://dx.doi.org/10.1090/S0002-9939-07-08810-7

Liu, W.-J., \& Krstić, M. (2002). Global boundary stabilization of the Korteweg-de VriesBurgers equation. Comput. Appl. Math., 21(1), 315-354. (Special issue in memory of Jacques-Louis Lions)

Marz, S., \& Cerpa, E. (2014). Output feedback control of the linear Korteweg-de Vries equation. IEEE Conference on Decision and Control.

Marx, S., \& Cerpa, E. (2016). Output feedback stabilization for the Korteweg-de Vries equation. arXiv:1609.06096v3 [math.AP]. Available from http://arxiv.org/abs/ 1609.06096 
Massarolo, C. P., Menzala, G. P., \& Pazoto, A. F. (2007). On the uniform decay for the Korteweg-de Vries equation with weak damping. Math. Methods Appl. Sci., 30(12), 1419-1435. Available from http://dx.doi.org/10.1002/mma.847

Rosier, L. (1997). Exact boundary controllability for the Korteweg-de Vries equation on a bounded domain. ESAIM Control Optim. Calc. Var., 2, 33-55. Available from http://dx.doi.org/10.1051/cocv: 1997102

Rosier, L. (2000). Exact boundary controllability for the linear Korteweg-de Vries equation on the half-line. SIAM J. Control Optim., 39(2), 331-351. Available from htt. IN dx.doi.org/10.1137/S0363012999353229

Rosier, L., \& Zhang, B.-Y. (2006). Global stabilization of the generalized Kortewsg-de Hies equation posed on a finite domain. SIAM J. Control Optim., 45(3), 927-956. Available from http://dx.doi.org/10.1137/050631409

Rosier, L., \& Zhang, B.-Y. (2009). Control and stabilization of the Korteweg de Vries equation: recent progresses. J. Syst. Sci. Complex., 22(4), 647-502. Available from http://dx.doi.org/10.1007/s11424-009-9194-2

Russell, D. L., \& Zhang, B. Y. (1996). Exact controllability arid stabilizability of the Korteweg-de Vries equation. Trans. Amer. Math. Soc. 348(9), 3643-3672. Available from http://dx.doi.org/10.1090/S0002-9947-96-015i2-8

Sakthivel, R. (2009). Robust stabilization the Korteweg-de Vries-B urgers equation by boundary control. Nonlinear Dynam., 58(4), 739-744. Available from http://dx.doi.org/ 10.1007/s11071-009-9514-z

Smaoui, N., El-Kadri, A., \& Zribi, M. (2010a). Adaptive boundary control of the forced generalized Korteweg-de Vries-Burgers equation. Eur. J. Control, 16(1), 72-84. Available from http://dx.doi.org/10.3166/ejc.15.72-84

Smaoui, N., El-Kadri, A., \& Zribi, M. (2010b). Nonlinear boundary control of the unforced generalized Korteweg-de Vries-Burgers equation. Nonlinear Dynam., 60(4), 561-574. Available from http://dx.doi.org:10.1007/s11071-009-9615-8

Zhang, B. Y. (1994). Boundary stabilization of the Korteweg-de Vries equation. In Control and estimation of distribuied purameter systems: nonlinear phenomena (Vorau, 1993) (Vol. 118, pp. 371-389). Birkï̈user, Basel.

Zhang, B.-Y. (1999). Exact botudary controllability of the Korteweg-de Vries equation. SIAM J. Control Optim., 37(2), 543-565. Available from http://dx.doi.org/10 $.1137 / \mathrm{S} 0363012997327501$ 


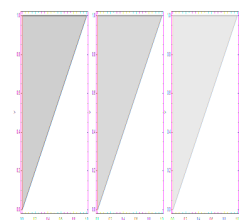

Figure 1. Triangular domain $T$ 


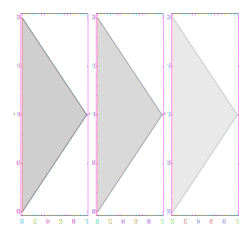

Figure 2. Triangular domain $T_{0}$ 


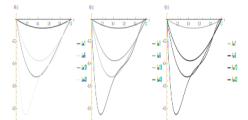

FiguRE 3. Control gain $k(0, y)$ for different values of $\lambda$ 


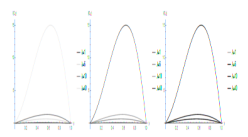

Figure 4. Inverse control gain $l(0, y)$ for different values of $\lambda$ 


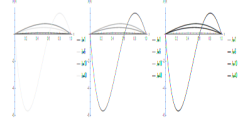

Figure 5. Control gain $p_{1}(x)$ for different values of $\lambda$

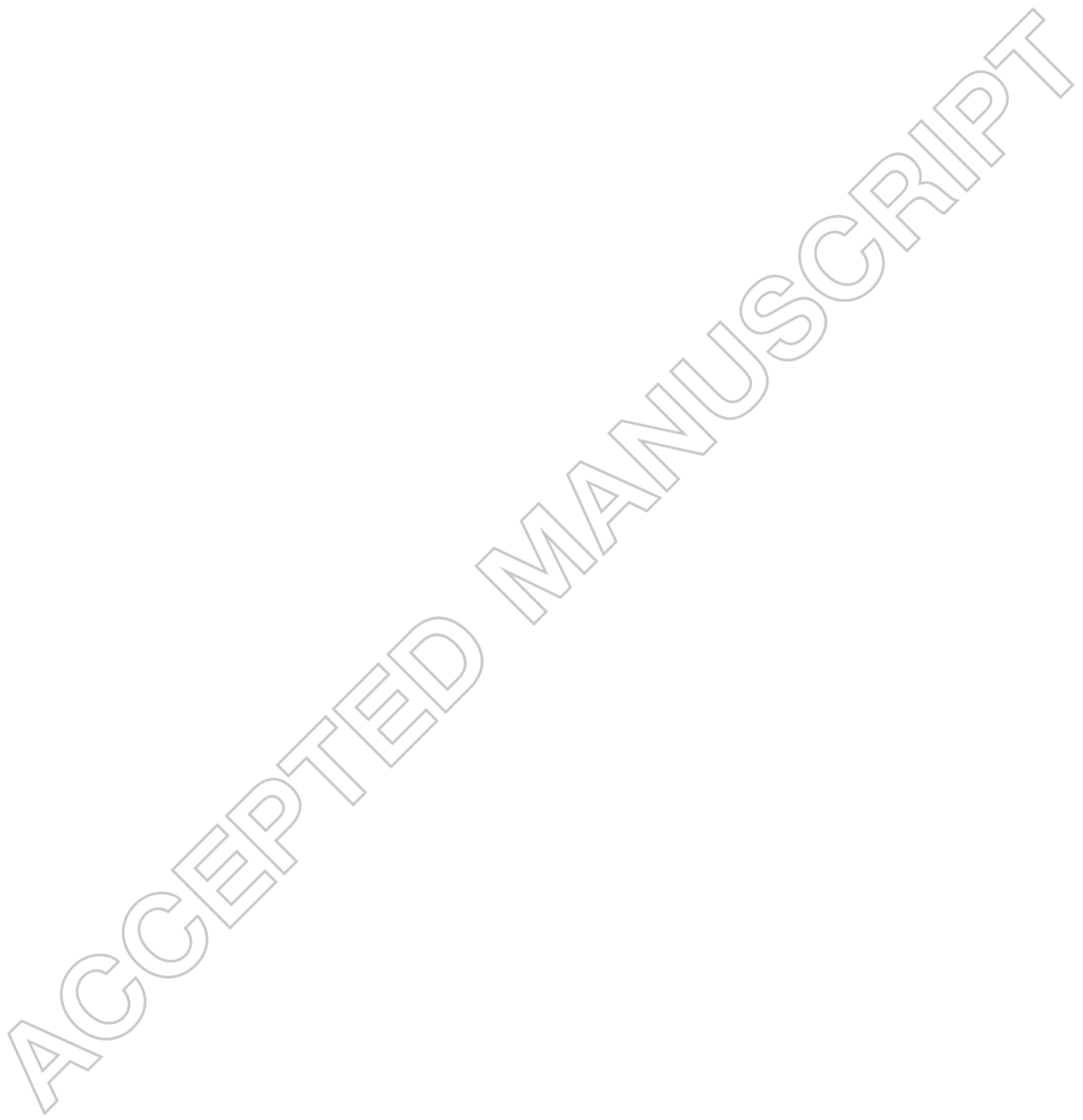




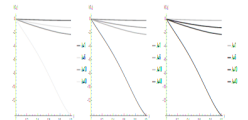

Figure 6. Control gain $k(0, y)$ for different values of $\lambda$ 


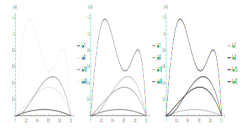

FiguRE 7. Control gain $p_{1}(x)$ for different values of $\lambda$ 


\begin{tabular}{|c|c|c|}
\hline$n$ & $\max _{(s, t) \in T_{0}}\left|H^{n}(s, t)\right|$ & $\sum_{k=1}^{n} \max _{(s, t) \in T_{0}}\left|H^{k}(s, t)\right|$ \\
\hline 1 & 0.11111 & 0.11111 \\
\hline 2 & 0.00538531 & 0.116496 \\
\hline 3 & 0.000831549 & 0.117327 \\
\hline 4 & 0.00010591 & 0.117433 \\
\hline 5 & $1.51134 \times 10^{-7}$ & 0.117433 \\
\hline 6 & $1.26128 \times 10^{-9}$ & 0.117433 \\
\hline 7 & $2.73963 \times 10^{-11}$ & 0.117433 \\
\hline 8 & $5.3179 \times 10^{-13}$ & 0.117433 \\
\hline 9 & $9.18326 \times 10^{-15}$ & 0.117433 \\
\hline 10 & $1.42747 \times 10^{-16}$ & 0.117433 \\
\hline
\end{tabular}

TABle 1. Numerical values demonstrating the fast con vergence $(\lambda=1)$ 